\title{
Advanced Methods to Enable 4D Imaging of Polymer Electrolyte Fuel Cells by X- ray Computed Tomography
}

\author{
Robin T. White ${ }^{1}$, Sebastian Eberhardt ${ }^{1}$, Yadvinder Singh $^{1}$, Dilip Ramani ${ }^{1}$, Francesco P. Orfino ${ }^{1}$, Monica \\ Dutta $^{2}$, and Erik Kjeang ${ }^{1}$ \\ 1. Fuel Cell Research Laboratory (FCReL), School of Mechatronic Systems Engineering, Simon Fraser \\ University, Surrey, Canada. \\ 2. Ballard Power Systems, Burnaby, Canada.
}

X-ray Computed Tomography (XCT) provides a unique ability to delve into the innermost structures through non-destructive imaging in diverse and extensive application areas. By combining the unique 3dimensional visualization through repeated identical location tomography scans at various temporal stages, powerful in-situ investigations of dynamic material properties can be obtained [1, 2]. This methodology is often termed as 4DCT and is well suited to study various dynamic and evolutionary processes in polymer electrolyte fuel cell (PEFC) systems. Detailed images of the membrane electrode assembly (MEA) can be periodically obtained while the cell is still assembled in its operational housing as well as while it is producing current [3].

The commercial viability of PEFCs has increased rapidly over recent years with applications in public/commercial transportation, back-up power, un-manned autonomous systems, and electronic devices $[4,5,6]$. PEFC MEAs are assembled as a series of layers, each having specific functionalities to optimize the cell performance during electrochemical conversion of chemical potential energy, in the way of hydrogen and oxygen, into useable electrical power, heat, and water. These layers consist of: (i) cathode and anode gas diffusion layers (GDL), made up of carbon fibres to allow electron conduction; (ii) cathode and anode catalyst layers, made up of platinum nanoparticles on carbon support surrounded by ionomer binder; and (iii) a centrally located perfluorinated membrane, which is electrically insulating, but acts as an ion bridge to allow the completion of the electrochemical reactions. These layers are surrounded by flow fields, which allow for the external gas supply of hydrogen and air; along with the electrical connections, gaskets and compression plates to seal the fuel cell. These PEFC components/materials can undergo considerable changes during operation and lifetime testing through critical degradation processes, which can be uniquely captured using 4DCT in this complex multilayered system.

Extraction of quantitative information regarding material/geometrical properties, such as thickness, porosity, saturation, and local composition, as well as variation in these properties and material morphology during any degradation process, is vastly facilitated by advanced image processing and visualization methods. In this work, custom tools and analysis methods are developed and allow for new insights into the temporal changes of water saturation [7], cathode catalyst layer morphology [3], and membrane crack propagation [8]. It has been found that during ageing, the morphological interaction between different layers can have a considerable impact on degradation mechanisms such as crack propagation. We also present an overview of the 4DCT approach applied to various fuel cell degradation studies, observing changes in material morphology by same location tracking during in situ and operando imaging (see Figure 1), as well as GDL water distribution during operando imaging. A custom workflow that allows for the segmentation of all MEA layers is also presented, as shown in 
Figure 2. These results uncover unique evidence around the strongly interactive nature of material degradation within a fuel cell that has been previously unobserved [9].

\section{References:}

[1] J. Kastner et al, 7th Conference on Industrial Computed Tomography, Leuven, Belgium, 2017. [2] J. Gelb, Adv. Mat. Proc. 170 (2012), p. 14.

[3] R. T. White et al, J. Power Sources 350 (2017), p. 94.

[4] "Markets," Ballard, 2018. [Online]. Available: http://ballard.com/markets. [Accessed 9 February 2018].

[5] "Transportation," Fuel Cell and Hydorgen Energy Association, [Online]. Available:

http://www.fchea.org/transportation. [Accessed 9 February 2018].

[6] A. Hoffrichter, in 12th International Hydrail Conference, Graz, 2017.

[7] S. Eberhardt et al, in ECS Meeting Abstracts, 2017.

[8] Y. Singh et al, in ECS Meeting Abstracts, 2017.

[9] Funding for this research was provided by the Natural Sciences and Engineering Research Council of Canada, Canada Foundation for Innovation, British Columbia Knowledge Development Fund, and Ballard Power Systems through an Automotive Partnership Canada grant.

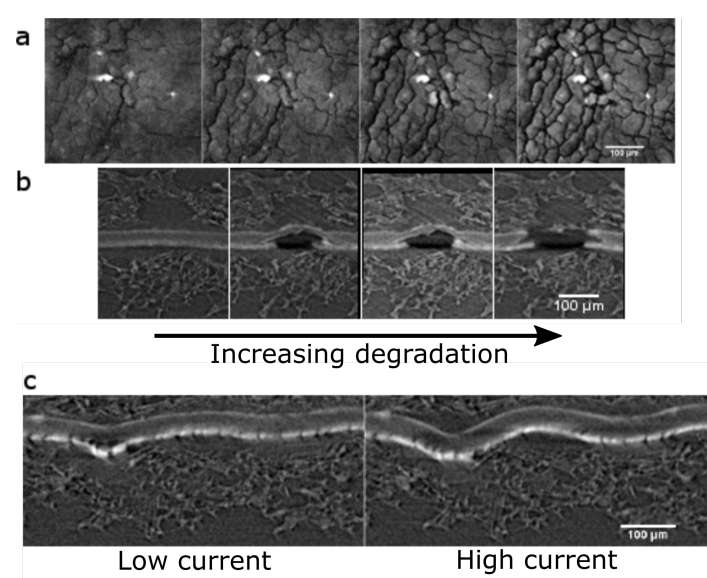

Figure 1. 4DCT of PEFC components following: a.) cathode catalyst layer degradation cycles; b.) mechanical stress cycles on membrane; and c.) swelling of membrane by water absorption after increased current density in cathode degraded cells

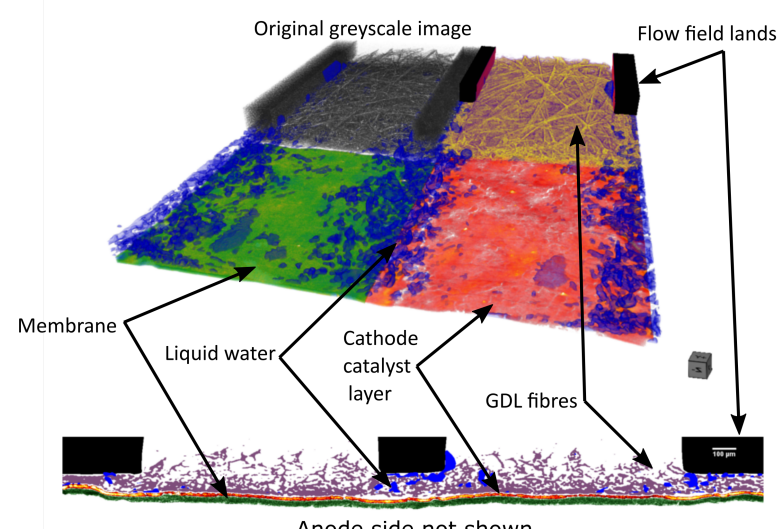

Figure 2. Full segmentation and visualization of all PEFC component layers and liquid water by advanced processing methods (anode side not shown) 